\section{Next Generation Programmable Bio-Nano- Chip System for On-Site Quantitative Drug Detection in Oral Fluids}

\section{Abstract}

Current on-site drug of abuse detection methods involve invasive sampling of blood and urine specimens, or collection of oral fluid, followed by qualitative screening tests using immunochromatographic cartridges. Test confirmation and quantitative assessment of a presumptive positive are then provided by remote laboratories, an inefficient and costly process decoupled from the initial sampling. Recently, a new noninvasive oral fluid sampling approach that is integrated with the chip-based Programmable Bio-Nano-Chip ( $p$-BNC) platform has been developed for the rapid ( 10 minutes), sensitive detection $(\sim \mathrm{ng} / \mathrm{ml}$ ) and quantitation of 12 drugs of abuse. Furthermore, the system can provide the time-course of select drug and metabolite profiles in oral fluids. For cocaine, we observed three slope components were correlated with cocaine-induced impairment using this chipbased $\mathrm{p}$-BNC detection modality. Thus, this $\mathrm{p}$-BNC has significant potential for roadside drug testing by law enforcement officers. Initial work reported on chipbased drug detection was completed using 'macro' or "chip in the lab" prototypes, that included metal encased "flow cells", external peristaltic pumps and a bench-top analyzer system instrumentation. We now describe the next generation miniaturized analyzer instrumentation along with customized disposables and sampling devices. These tools will offer real-time oral fluid drug monitoring capabilities, to be used for roadside drug testing as well as testing in clinical settings as a non-invasive, quantitative, accurate and sensitive tool to verify patient adherence to treatment.

Keywords: Drugs of abuse; Programmable Bio-nano-chip; Oral fluid, Multiplexed; On-site testing

Received: October 02, 2015, Accepted: November 16, 2015, Published: November 23, 2015

\section{Introduction}

In 2013, 21.6 million Americans were dependent on drugs or were drug abusers, representing $8.2 \%$ of our population aged 12 and over. While most of these individuals had problems with only alcohol, 2.6 million has problems with illicit drugs and alcohol, and 4.3 million with illicit drugs alone (https:// d14rmgtrwzf5a.cloudfront.net/sites/default/files/resource_ guide.pdf). Marijuana is the primary illicit drug with 4.2 million abusers or dependents, followed by pain relievers (1.9 million), cocaine $(855,000)$ and heroin $(517,000)$ (www.drugabuse.gov/ publications/drugfacts/nationwide-trends). Unfortunately, of the 21.6 million Americans who need substance use treatment, 20.2 million of them did not receive it (Results from the 2013 National Survey on Drug Use and Health: Summary of National Findings.

\section{Nicolaos Christodoulides ${ }^{1,2}$, Richard De La Garza III-6, Glennon W Simmons ${ }^{1,2}$, Michael P McRae", Jorge Wong ${ }^{1,2}$, Thomas F Newton ${ }^{3,4,6}$, Thomas R. Kosten ${ }^{3-6}$, Ahmed Haque' and John T McDevitt ${ }^{1,2,7}$}

\section{Department of Bioengineering, Rice University, Houston TX, USA \\ 2 Department of Chemistry, Rice University, Houston TX, USA \\ 3 Menninger Department of Psychiatry \& Behavioral Sciences, Baylor College of Medicine, Houston TX, USA \\ 4 Department of Pharmacology, Baylor College of Medicine, Houston TX, USA \\ 5 Department of Neuroscience, Baylor College of Medicine, Houston TX, USA \\ 6 Department of Veterans Affairs Medical Center, Houston, TX, USA \\ 7 Department of Biomaterials, Bioengineering Institute, New York University, New York, NY, USA}

Corresponding author: John T McDevitt

$\equiv$ mcdevitt@nyu.edu

Department of Biomaterials, Bioengineering Institute, New York University, 433 First Avenue, Room 820, New York, NY 10010-4086, USA

Tel: 212-998-9204

Citation: Christodoulides N, De La Garza R, Simmons GW, et al. Next Generation Programmable Bio-Nano-Chip System for On-Site Quantitative Drug Detection in Oral Fluids. J Drug Abuse. 2015, 1:1.

Rockville, MD: Substance Abuse and Mental Health Services Administration; 2014 September 2014.). Furthermore, in the US each year drug abuse and drug addiction cost employers over 
\$122 billion in lost productivity time and another $\$ 15$ billion in health insurance costs [1]. Routine screening for substance use disorders could alter this statistic and get more people the help they need.

Since the inception of medication-assisted treatment (MAT) for drug addiction, drug testing has provided both an objective measure of treatment efficacy and a tool to monitor patient progress. Likewise, increasing emphasis on treatment outcomes as evidence of program effectiveness has added significance to drug tests in Treatment Programs (TPs), as well as use of drug test results in response to quality assurance requirements.

However, up to this juncture, drug testing in treatment settings has been limited to either remote drug testing via laboratoryconfined procedures (LC-MS/MS or ELISA) associated with long turn-around time for results reporting or on-site urine testing via immunochromatographic test strips (ICS), which are known to have limited multiplexing and semi-quantitative measurement capacities.

Indeed, there is a need for an advanced on site drug testing solution to be implemented at addiction treatment settings. Drug test results help policymakers and TP administrators detect and monitor emerging trends in substance abuse that may signal a need to redirect resources. Drug use patterns have changed markedly in recent decades; for example, benzodiazepines, amphetamines, methamphetamine, and cocaine have increased in popularity while barbiturate use has diminished. New substances of abuse or combinations of substances and methods of ingestion present new treatment challenges and funding concerns (Center for Substance Abuse Treatment. MedicationAssisted Treatment for Opioid Addiction in Opioid Treatment Programs. Rockville (MD): Substance Abuse and Mental Health Services Administration (US); 2005. (Treatment Improvement Protocol (TIP) Series, No. 43.) Chapter 9. Drug Testing as a Tool. Available from: http://www.ncbi.nlm.nih.gov/books/NBK64151).

Even though significant progress has been made towards the development of lab-on-a-chip (LOC) devices over the years serving a variety of applications, [2-5] few of these devices have obtained widespread commercial and clinical adoption [6]; likewise, integration of microfluidic and instrumentation components into unified system remains a central challenge in this area, as well as is the creation of an automated workflow compatible for nonexperts with limited to no human intervention required between sample introduction and data presentation [7].

For the past decade, the McDevitt Research Group has been focused on the development of the p-BNC, a novel chip-based sensor that provides rapid, quantitative, and multiplexed biomarker assessments for clinical diagnostic applications including heart disease, oral cancer, ovarian cancer, trauma, and many more health conditions [8-11]. By utilizing the principles of microfluidics and a lab-on-a-chip approach, the p-BNC assays provide a way for monitoring multiple biomarkers simultaneously, require drastically reduced volumes of chemical reagents, and can provide biomarker diagnosis in minutes as compared to the week-long wait times of market available lab-based tests.

Herein provided is an update on the further development of the bead-based Programmable Bio-Nano-Chip ( $p-B N C)$ drug tests reported recently by Christodoulides et al. [12]. It is expected that this next generation of a microfluidics point-of-care (POC) solution, including a cartridge and miniaturized analyzer, will permit real-time screening of drug users in treatment facilities.

\section{Material studied, area descriptions, methods, techniques}

As described previously by Christodoulides et al. [12], the beadbased $\mathrm{p}$-BNC drug tests function with non-invasive oral fluid sampling; more specifically, the process involves use of an oral swab to brush the entire upper and lower gum line, and then insertion of the swab into a specimen collection tube to extract the sample into the assay fluid that includes the tracer antibody used in bead/chip-based competitive immunoassay. The p-BNC reveals if there are specific drugs (i.e., drug immune-equivalents) present in the body within $\sim 10$ minutes. In this setup, the test is initiated with the mixing of the salivary sample with the tracer. The mixture is then delivered to the $\mathrm{p}$-BNC flow cell equipped with a bead-array platform. The test sequence is completed with a final washing step with PBS. In the absence of drug in the sample, the tracer antibody specifically recognizes its corresponding drug sensor(s) and, thereby, produces a strong signal on the surface, as well as within the interior of the porous bead in the array, while in the presence of drug in the sample the binding of the in a drugspecific, dose-dependent manner.

\section{Results and Discussion}

The technology described here is a complete drug testing assay solution (Figure 1), executed within a microfluidics point-ofcare $(\mathrm{POC})$ cartridge, in conjunction with miniaturized analyzer with proven multiplexed testing capacity (Figure 2) for real-time screening of major drugs of abuse (Table 1 ) in oral fluids.

A key feature differentiates the $p$-BNC from other analytical schemata. Unlike the typical microfluidic, biochip and lab-on-achip (LOC) paradigms, in which a single 'chip' is created specific to a type of cell/protein/oligo, the p-BNC is able to measure a broad portfolio of analytes within the same compact system. We can easily create targeted panels by including modular

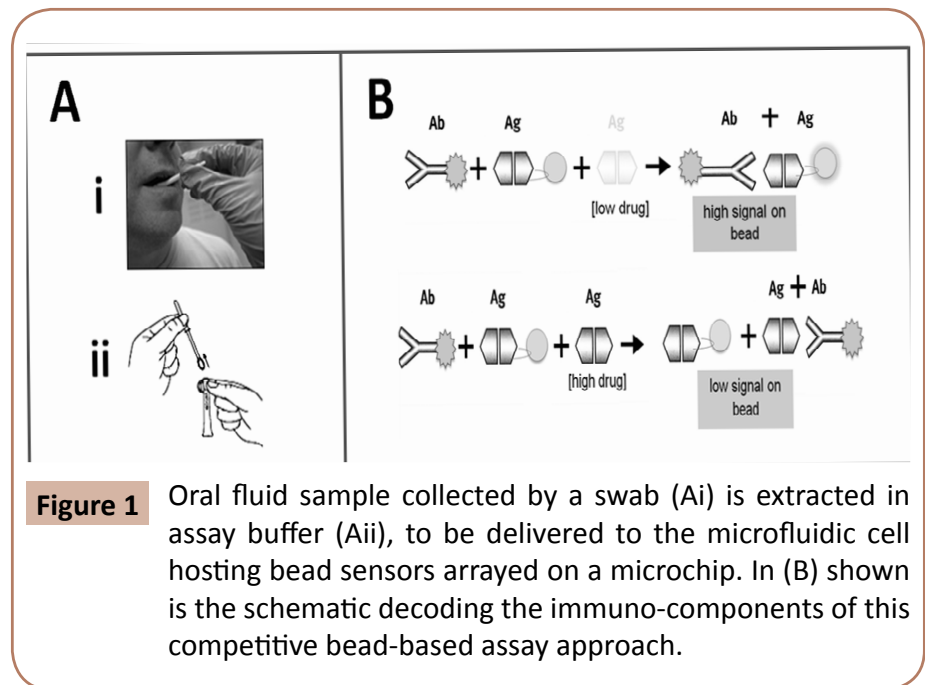




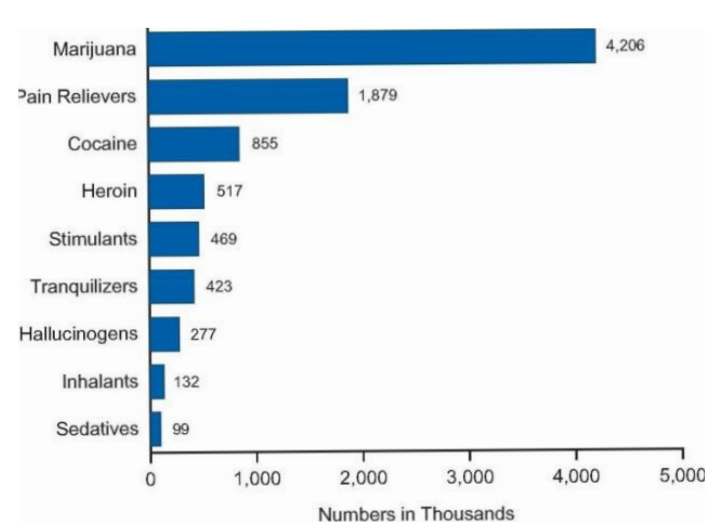

Figure 2 Specific illicit drug dependence or abuse in the past year among persons aged 12 or older in 2013.

Table 1 The $\mathrm{p}$-BNC-based tests for drugs of abuse and their limits of detection (LOD) and quantitation (LOQ) as acquired by the 'macro' laboratory prototype.

\begin{tabular}{|c|c|}
\hline DRUG & LOD/LOQ* $(\mathrm{ng} / \mathrm{mL})$ \\
\hline Tetrahydrocannabinol (THC) & $1.0 / 5$ \\
\hline Morphine & $0.6 / 2$ \\
\hline Amphetamine & $0.22 / 1$ \\
\hline Methamphetamine & $1.0 / 8.6$ \\
\hline Cocaine/benzoylecgonine (BZE) & $1.3 / 10$ \\
\hline 3,4-methylenedioxyamphetamine (MDA) & $6.7 / 10$ \\
\hline 3,4-methylenedioxy-N-methylamphetamine & $1.5 / 3.6$ \\
\hline (MDMA) & $1.3 / 5$ \\
\hline Methadone & $0.14 / 1$ \\
\hline Diazepam & $1.0 / 10$ \\
\hline Oxazepam & $3.8 / 30$ \\
\hline Temazepam & $7.4 / 20$ \\
\hline Nordiazepam & \\
\hline
\end{tabular}

*Assay performance characteristics shown are those of single-analyte tests on the 'macro' laboratory $\mathrm{p}$-BNC device.

reagent packages, bead capture elements, and size-tuned cell collection ensembles. Another important distinction of the p-BNC technology is the different mechanism of analyte capture. While most microfluidic approaches employ planar arrays, the p-BNC uses high surface area 3D beads that efficiently concentrate various analytes from biofluids. The technology displays results in minutes, which makes them available at the point-of-need.

Figures $3 \mathrm{~A}$ and $3 \mathrm{~B}$ illustrate the various stages of evolution of the two major components of this technology, lab card and portable analyzer, respectively. It should be noted, the majority of the initial development work on the chip-based drug detection already reported was completed using 'macro' laboratory prototypes, that included metal encased "flow cells" and bench-top instrumentation [12]. At the same time, parallel efforts have been directed toward the establishment of portable 'micro' instrumentation [13] and disposable labcards that now may be used at the point of need settings.

The fully integrated p-BNC employs the compact labcard (Figure 4A), which condenses the performance features across many size regimes: nanometer-scale antibodies and micrometer-sized beads operate in tandem with the millimeter-scale injection-
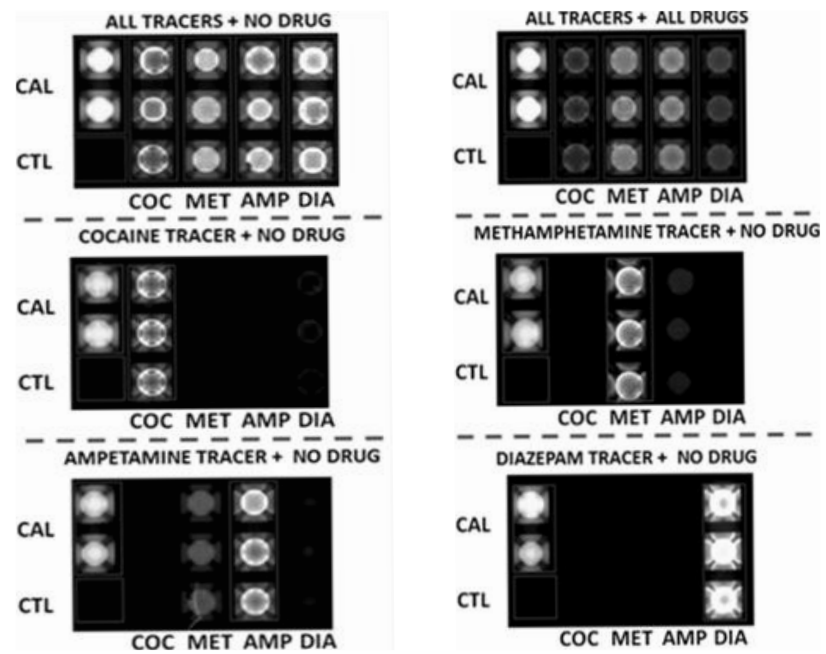

Figure 3 Multiplexed drug-specific $p$-BNC panel recorded with the 'macro' laboratory prototype. Demonstration of the specificity of tracers for their respective drug targets.

CAL: beads coupled to a fixed amount of fluor serve as calibrators of the $p$-BNC system

CTL: beads coupled to bovine serum albumin alone, serve as negative controls and further indicators of the specificity of the reactions that take place within the flow cell.

molded plastic support structure. The labcard also holds solidphase reagents, a waste reservoir and a built-in metering capacity. Blister packs contain liquid-phase reagents, and linear actuators control fluid flow, thus simplifying the "world-to-chip" interface for ultimate usability at the point-of-care. Using inexpensive plastic materials for labcard construction along with similarly cost-effective agarose beads, the integrated structure has the potential to serve as an accessible and flexible bioassay system.

Each disposable labcard is used with a single patient sample. After a specimen is collected and introduced into the compact p-BNC, the labcard is inserted into the analyzer (Figure 4B). Reagents from the blister packs flow through the fluidic cartridges of the labcard and the assay is performed. After data acquisition, the labcard is ejected, and the standalone reader is ready for another labcard. Critical to ease and cost, both the labcard and analyzer use the same fluid, light and waste handling design; the core of the $\mathrm{p}-\mathrm{BNC}$ is thus universal to a broad class of analytes.

Likewise, the $\mathrm{p}$-BNC is an injection-molded cartridge with overall credit card sized dimensions. It comprises a network of fluidic components that ensure the complete transfer and process of a blood or saliva sample to generate quantitative cellular, genomic or proteomic information. Shown in Figure $\mathbf{4 A}$ is a representation of the multiple biochip functions that serve to eliminate some of the constraints imposed by traditional laboratory methods. The sample introduction requirements are compatible with minimally invasive (pin prick) or non-invasive (saliva) specimen. Reagents are stored dry on a conjugate pad embedded within the biochip, and are dissolved as needed, through pre-packed buffer (PBS) contained in biochip-integrated pouches. Here, all 
A.
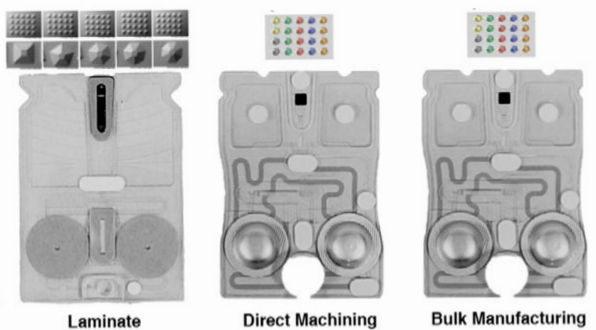

Flow Cell

Laminate

Direct Machining

Bulk Manufacturing

B.

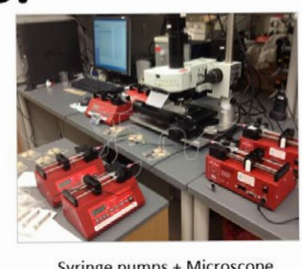

Syringe pumps + Microscope

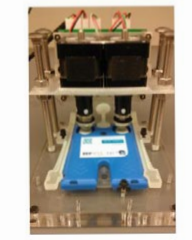

Blister + Mechanical Actuation

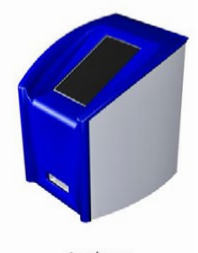

Analyzer

Figure 4 Pathway towards complete p-BNC integration. Top panel (A) illustrates the evolution of $p$-BNC disposables, from re-usable flow cells to single use lab cards or cartridges. The first iteration, the metal encased flow cell, included a silicon microchip hosting the array of bead sensors for the assay, and functioned in conjunction with external pumps and a bench-top customized fluorescent microscope. Rapid prototyping (design to parts in a few hours) using relatively inexpensive infrastructure allowed the generation and optimization of the next generation laminate lab cards, which in turn provided the specifications for direct machining. Once the most optimal design of lab cards was identified and validated, injection molding was applied as a means for mass scalable production. Evolution towards a portable analyzer system (Bottom panel- B) involved a 3-step process, incepting with the use of syringe pumps and a microscope, replacement of pumps with blisters and mechanical actuation with external devices and, finally, the portable analyzer, a completely integrated solution.

processing steps are conducted within the microfluidic network of the biochip via actuation mechanisms within the analyzer without human intervention. These features eliminate the need for external fluidics such as pumps, tubing, and connectors. The sample is directed to an on-chip waste reservoir, which provides a safe containment of bio-hazardous fluids. The entire biochip can be discarded as solid waste after the assay, facilitating biohazard waste management. Therefore, this integrated system has the potential to reduce cost and reduce the risk for leaks and contamination. Collectively, these essential features serve to facilitate the transition from chips-in-the-lab to labs-on-achip, and offer significant opportunities for point-of-care needs and, specifically in this project, for the proposed point-of-need application (Figure 5).

\section{Conclusion}

The new efforts herein described place into the pipeline new options for drug testing that have significant potential to influence measurement of drug samples for a wide variety of settings.
A.

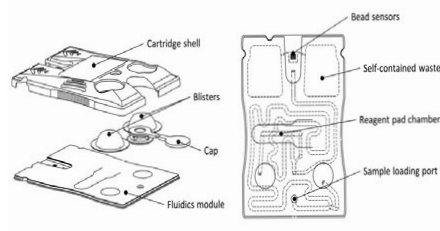

B.

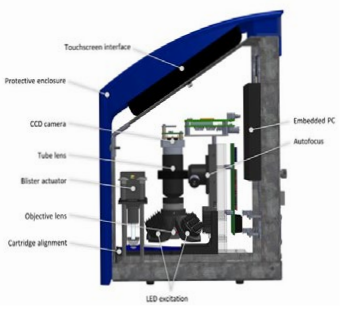

Figure 5 Credit card-sized p-BNCs and portable analyzer may serve the needs of a typical laboratory-based infrastructure at the point-of-need.

Up to this juncture, 12 drugs have been targeted by p-BNC (Table 1). The novelty of the proposed $p$-BNC drug testing solution for TPs relates to the following: The $p$-BNC drug testing platform compares favorably to those of LC-MS/MS and ICS counterparts, considered as the current gold standards (or reference methods) for the laboratory and the point-of-need settings, respectively (Table 2). Unlike ICS tests, which are qualitative (Yes/No) type of tests, both LC-MS/MS and p-BNC-based drug tests are fully quantitative, albeit $\mathrm{p}$-BNC tests are highly dependent on the specificity of the antibody reagents that they employ. Likewise, with exceptional limits of detection, ranging from 0.14 to 7.4 $\mathrm{ng} / \mathrm{mL}$, the majority of the $\mathrm{p}$-BNC tests exhibit a capacity to detect as low concentrations of the drugs as those measurable by LC-MS/MS and, for the most part, lower amounts than those detectable in oral fluids than their ICS test counterparts. Furthermore, unlike the laboratory-confined LC-MS/MS, p-BNC tests require no tedious and time-consuming sample processing, and they are amenable to settings outside the laboratory, while still offering the capacity to multiplex, or to test for more than one analyte (drug) concurrently using microliters of sample.

The utility of the p-BNC method is mostly directed towards quantitation of the drug to which the patient is addicted to, as a means to monitor and confirm abstinence and treatment efficacy. The ability to also monitor levels of the drug used for treatment presents itself as an additional tool for managing dosage. The ease of use, rapid test results, low cost/test and accuracy/precision associated with p-BNC measurements promise to allow drug tests in the TP setting to be performed with sufficient frequency and randomness to assist in making informed decisions about take-home privileges and assessment of patient's response to treatment. Furthermore, conforming to Federal and State regulations, the p-BNC will allow for frequency of testing to be appropriate for each patient and should allow for a caring and rapid response to possible relapse.

\section{Acknowledgement}

Support for the $\mathrm{p}$-BNC core instrumentation work was provided also by the National Institutes of Health $(\mathrm{NIH})$ through the National Institute of Dental and Craniofacial Research (Award Number 5 U01 DE017793).

Funding support for this work was provided by the United 
Table 2 Advantages of $p$-BNC over existing methods for the quantitative determination of drugs of abuse in a narcotics treatment facility.

\begin{tabular}{|cccccccc|} 
Method & $\begin{array}{c}\text { Sample } \\
\text { Processing }\end{array}$ & $\begin{array}{c}\text { Quick } \\
\text { TAT* }\end{array}$ & $\begin{array}{c}\text { Multiplexing } \\
\text { Capacity }\end{array}$ & Quantitative & Sensitivity & $\begin{array}{c}\text { Amenable } \\
\text { to Point } \\
\text { of Care }\end{array}$ \\
\hline & TEDIOUS & NO & YES & YES & HIGH & NO \\
\hline & EASY & YES & LIMITED & NO & LIMITED & YES \\
\hline & EASY & YES & YES & YES & HIGH & YES \\
\hline
\end{tabular}

Kingdom (UK) Home Office Centre of Applied Science and Technology (CAST). This sponsor was involved in the design of the study and contributed suggestions on the interpretation of data and definition of the next steps.

Part of this work is the result of work facilitated with resources and the use of the infrastructure at the Michael E. DeBakey VA Medical Center, Houston, Texas.

\section{Conflict of Interest}

Principal Investigator, John T. McDevitt, has an equity interest in SensoDX, LLC. Inc, and also serves on Scientific Advisory Board. The terms of this arrangement have been reviewed by Rice University and by New York University and he is currently under a management plan in accordance with their conflict of interest policies. 


\section{References}

1 Miller T, Hendrie D (2008) Substance Abuse Prevention Dollars and Cents: A Cost-Benefit Analysis, DHHS Pub. No. (SMA) 07-4298. Rockville, MD: Center for Substance Abuse Prevention, Substance Abuse and Mental Health Services Administration.

2 Chin CD, Linder V, Sia SK (2007) Lab-on-a-chip devices for global health: past studies and future opportunities. Lab Chip 7: 41-57.

3 Walt DR (2005) Chemistry. Miniature analytical methods for medical diagnostics. Science 308: 217-219.

4 Yager P, Edwards T, Fu E, Helton K, Nelson K, et al. (2006) Microfluidic diagnostic technologies for global public health. Nature 442: 412-418.

5 Bissonnette L, Bergeron MG (2010) Diagnosing infections--current and anticipated technologies for point-of-care diagnostics and home-based testing. Clin Microbiol Infect 16: 1044-1053.

6 Whitesides GM (2006) The origins and the future of microfluidics. Nature 442: 368-373.

7 Kelley SO, Mirkin CA, Walt DR, Ismagilov RF, Toner M, et al. (2014) Advancing the speed, sensitivity and accuracy of biomolecular detection using multi-length-scale engineering. Nat Nanotechnol 9: 969-980.
8 Christodoulides N, Tran M, Floriano PN, Rodriguez M, Goodey A, et al. (2002) A microchip-based multianalyte assay system for the assessment of cardiac risk. Anal Chem 74: 3030-3036.

9 Christodoulides N, Floriano PN, Sanchez X, Li L, Hocquard K, et al. (2012) Programmable bio-nanochip technology for the diagnosis of cardiovascular disease at the point of care. Methodist Debakey Cardiovasc J 1: 6-12.

10 Floriano PN, Christodoulides N, Miller CS, Ebersole JL, Spertus J, et al. (2009) Use of saliva-based nano-biochip tests for acute myocardial infarction at the point of care: a feasibility study. Clin Chem 55: 1530-1538.

11 Raamanathan A, Simmons GW, Christodoulides N, Floriano PN, Furmaga WB, et al. (2012) Programmable bio-nano-chip systems for serum CA125 quantification: toward ovarian cancer diagnostics at the point-of-care. Cancer Prev Res (Phila) 5: 706-716.

12 Christodoulides N, De La Garza R II, Simmons GW, McRae MP, Wong $\mathrm{J}$, et al. (2015) Application of Programmable Bio-Nano-Chip System for the Quantitative Detection of Drugs of Abuse in Oral Fluids. Drug Alcohol Depend 153: 306-313.

13 McRae MP, Simmons GW, Wong J, Shadfan B, Gopalkrishnan S, et al. (2015) Programmable bio-nano-chip system: a flexible pointof-care platform for bioscience and clinical measurements. Lab Chip 15: 4020-4031. 